\title{
DEVELOPMENT OF A PULSED HIGH MAGNETIC FIELD LABORATORY AT HUAZHONG UNIVERSITY OF SCIENCE AND TECHNOLOGY
}

\author{
Cheng Wang, Huazhong University of Science and Technology, Wuhan, China \\ Xian Zhong Duan, Huazhong University of Science and Technology, Wuhan, \\ China \\ Ke Xun Yu, Huazhong University of Science and Technology, Wuhan, China \\ Tao Peng, Huazhong University of Science and Technology, Wuhan, China \\ Zheng Cai Xia, Huazhong University of Science and Technology, Wuhan, China \\ Hong Fa Ding, Huazhong University of Science and Technology, Wuhan, China \\ Liang Li, Huazhong University of Science and Technology, Wuhan, China \\ GE Global Research Center, Niskayuna, USA \\ Fritz Herlach, Institute for Nanoscale Physics and Chemistry, K.U.Leuven, \\ Belgium \\ Johan Vanacken, Institute for Nanoscale Physics and Chemistry, K.U.Leuven, \\ Belgium
}

\begin{abstract}
A pulsed high magnetic field laboratory is to be funded and established at Huazhong University of Science and Technology (HUST), Wuhan, China by the Chinese Development and Reform Committee. In order to cover a wide spectrum of the experimental possibilities, the pulsed field coils are planned in the range of 50-80 $\mathrm{T}$ with pulse durations from $20 \mathrm{~ms}$ to $1000 \mathrm{~ms}$. Experience in the construction of the pulsed power supplies, pulsed magnet and the experimental equipment has been gained from a pilot laboratory at HUST since 2002. The lab building and the installation of a $1 \mathrm{MJ}, 25$ $\mathrm{kV}$ capacitor bank and the 100 MVA pulse generator have been completed. Two pulsed magnets with a $18 \mathrm{~mm}$ bore were made at K.U.Leuven in the context of the bilateral project between two universities. The magnets have been tested to $42 \mathrm{~T}$ and installed in two measuring stations. As the first experiment, the magneto-resistance of the high temperature superconductor $\mathrm{La}_{2-\mathrm{x}} \mathrm{Sr}_{\mathrm{x}} \mathrm{CuO}_{4}$ was measured.
\end{abstract}

\section{INTRODUCTION}

The construction of the pulsed high magnetic field facilities at the Huazhong University of Science and Technology started in 2002 in response to the demand for high fields in China. The laboratory is financed by the Ministry of Education. Meanwhile, HUST and K. U. Leuven applied for a bilateral project Flanders-China BIL02/01 for pulsed field development in 2002. In 2004, with the help of the pulsed field group at K.U.Leuven, the first magnet built at K.U.Leuven was tested at HUST with a small capacitor bank. In the spring of 2006, the construction of the pilot laboratory at HUST was basically finished and the first experiments were carried out. Based on experience with respect to safety, cryostat design, electrical diagnostics and optical signal transmission, the enhancement of the laboratory is now in progress. 


\section{THE LABORATORY}

Figure 1 shows the layout of the laboratory. The capacitor bank is laid at the corner of the experimental hall. On the right hand side of the capacitor bank, two measuring stations are built. The measuring stations are encaged in two separated steel-wall rooms, which can protect the facility from the coil explosion. Between the measuring stations and the control center, the control signal is transmitted by optical fibre, and the audio and video signals are transmitted by wireless devices. For the Local Area Network (LAN) and data communication, special photoelectric transformators are used instead of standard optical Ethernet, which makes the cost much lower. The offices and sample preparation room are located on the other side of the laboratory. The $100 \mathrm{MJ}, 100 \mathrm{MVA}$ pulsed generator is installed in between. The generator is planned to energize a $50 \mathrm{~T}$ flat-top pulse coil, which is planned to be built in the new project.

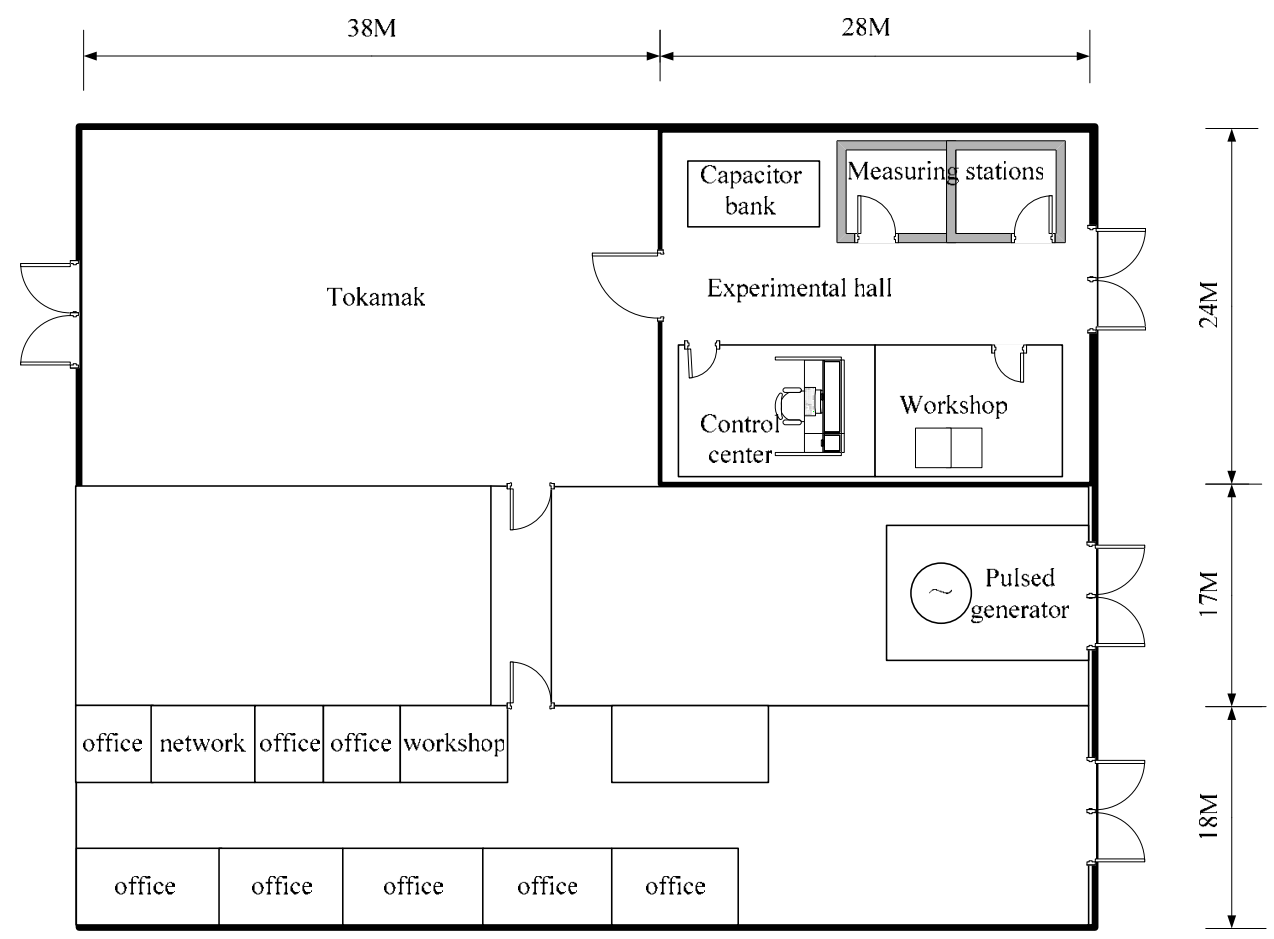

Figure 1. Layout of the pulsed field laboratory at HUST. The laboratory shares the building with a Tokamak installation. The capacitor bank, measuring stations, control room and a small workshop are arranged in the experimental hall. The offices are located on the other side of the building.

\section{CAPACITOR BANK}

The simplified scheme of the capacitor bank is given in Fig. 2. The capacitor bank consists of 58 capacitors with $55 \mu \mathrm{F}$ each. In order to protect the capacitor bank against short circuit, each capacitor is connected in series with an inductor and a fuse. A diode 
crowbar circuit is connected across the capacitor bank. The crowbar resistance can be adjusted from $0.09 \Omega$ to $0.54 \Omega$ with a step of $0.09 \Omega$ to modify the trailing edge of the field pulse and to minimize the heating of the coil. In the discharge circuit, a mechanical vacuum switch is used with the capability to support a maximum current of $30 \mathrm{kA}$. The capacitor bank can be discharged by a water resistor with a long time constant ( 1 minute), switched by a mechanical switch.

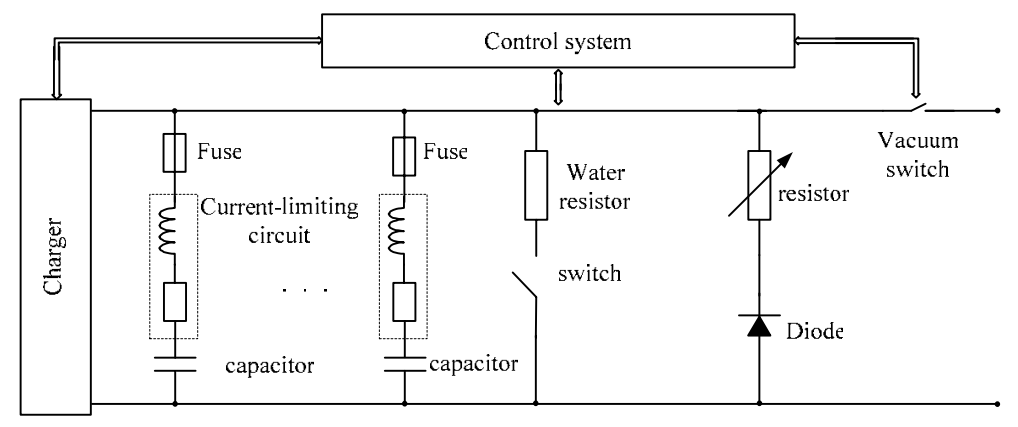

Figure 2. Simplified scheme of the capacitor bank.

In order to isolate the high voltage from the control center, an optical trigger signal produced in the control center is transmitted to the capacitor bank; and the signal is converted into an electric signal that triggers the switch. Presently, the system is grounded at the inner side of the magnet. The capacitor bank cannot be charged to negative voltage, and for the time being there are no provisions for reversing the field polarity. The specifications of the capacitor bank are:

- High energy density capacitors,

- $3.2 \mathrm{mF} / 25 \mathrm{kV} / 1 \mathrm{MJ}$,

- Charge voltage is reproducible within $0.5 \%$,

- Constant charging current (1 A) switching to constant power (40 kW),

- Charging time is less than 60 seconds.

\section{PULSED MAGNET DESIGN SOFTWARE}

The design of pulsed magnets involves the calculation of the stress distribution, the pulse shape and the temperature increase. The design process is aimed at the optimization of performance with respect to the desired characteristics of the pulsed field. Presently, two types of design tools are used for pulsed magnets. One is commercial finite element codes like ANSYS or Femlab ${ }^{1-2}$ which can calculate with more detail at the expense of modeling and computing time. The other is the simple one-dimensional computer code which can calculate the stresses and temperature distribution in the mid-plane of the coil with less time and more ease. To this end, the enhanced new code, Pulsed Magnet Design Software (PMDS2) has been developed in the context of the bilateral project between HUST and K.U.Leuven ${ }^{3-4}$.

The interface and operation of PMDS2 is quite similar to the first code, PMDS1, but the kernels are completely different. The engine of PMDS1 was developed with Fortran and the shell was written in Visual Basic $3.0^{5-6}$. PMDS1 solves the coupled differential equations for the stress distribution by matrix inversion; current distribution and coil heating are calculated by solving the differential equations for the diffusion of magnetic 
flux. The calculation is incremental: the field is increased in small steps and the equations are adapted when plastic deformation is encountered. PMDS2 is developed with Visual Basic 6.0. The stresses are calculated with total strain theory. The temperature is calculated with the eddy current approximation for the skin effect. The anisotropy of fibre composites is taken into account, including a database that can be easily adapted to include new data items as they are needed and become available. This code allows calculation of dual coil systems and the effect of a conducting cylinder. The main features of PMDS2 are listed below:

- Calculate the stress and heating in the mid-plane of the coil

- Design coils with up to 10 different types each of wire and reinforcement

- Design a split coil with axial gap in the mid-plane

- Design coils with axial concentric cooling channel

- Design foil coil

- Calculate either a monolithic coil incorporating a conducting cylinder or a dual coil system

- Specify the initial temperature for the discharge calculation

- Specify a background field for stress calculation

- Make recognizable .log files for ANSYS

- Take into account the anisotropy of fibre composites

- Material properties are stored in a convenient and flexible Excel database

- All relevant data of each coil design are stored in a standardized file

PMDS2 is user-friendly and efficient. It takes only a few minutes to define a coil and only a few mouse clicks to change parameters; calculating all the stresses, field distribution and pulse waveform for a 30-layer coil takes less than one minute. The results obtained from PMDS2 agree well with those obtained from experiments and commercial finite element software. For exploratory work and for the design of compact coils, PMDS2 is adequate and much more convenient. It has been adopted as a design platform in the European pulsed field project DeNUF ${ }^{7}$.

\section{COILS}

Presently, two pulsed magnets have been designed and manufactured according to the scheme developed at K.U.Leuven. These coils have internal reinforcement by fibre composites of variable thickness such that the stress is evenly distributed over the entire coil. External reinforcement is provided by a carbon fibre composite ${ }^{8}$. This scheme combines superior efficiency with ease of construction. The physical attributes of the two coils are outlined in Table 1.

Table 1. Physical Attributes of the Present Coils

\begin{tabular}{lcc}
\hline & Coil \#1 & Coil \#2 \\
\hline Design Field (T) & 70 & 50 \\
Bore (mm) & 18 & 18 \\
Pulse Duration (ms) & 15 & 40 \\
Height (mm) & 80 & 180 \\
Conductor Material & $\mathrm{Cu}$ & $\mathrm{Cu}$ \\
Reinforcement Material & Zylon + Glass + Carbon & Glass + Carbon \\
Impregnation & Wet-winding + vacuum & vacuum \\
\hline
\end{tabular}


The inner section of Coil \#1 is reinforced with Zylon fibre. Since Zylon cannot be well impregnated with vacuum impregnation, the wet-winding method was used. The outer section of Coil \#1 was reinforced with glass-fiber and impregnated with flow-through vacuum impregnation. Coil \#2 was reinforced entirely with glass-fiber, except for the outer reinforcing shell. This coil was vacuum-impregnated. During the impregnation procedure, ten hours after the epoxy went into the coil from the bottom flange, only a few drops of epoxy could be seen on the top flange of the coil. It seemed that the epoxy didn't go through the entire coil. Then the coil was impregnated from the top. The procedure was: we first poured some epoxy into the cylinder shell from the top of the coil and vacuum the coil and epoxy. After all the bubbles came out from the epoxy, a 2 bar pressure was applied on the epoxy. Then the epoxy went into the coil from the top and the glass-fibre was impregnated. In K.U.Leuven, many coils were successfully made with glass-fibre and vacuum impregnation. The reason why vacuum impregnation didn't work well for Coil \#2 was that the coil was too long. In the test, coil \#2 survived at $10 \mathrm{kV}$ voltage; testing at higher voltage is under way.

\section{DATA RECORDING SYSTEM}

The first experiments were based on the measurement of magneto-resistance on the high temperature superconductor $\mathrm{La}_{2-\mathrm{x}} \mathrm{Sr}_{\mathrm{x}} \mathrm{CuO}_{4}(\mathrm{x}=0.13)$. The layout of the data recording system is shown in Fig. 3. The system is based on an industrial computer equipped with a PCI card. Before the signal goes into the PCI card, it is amplified by a pre-amplifier. Between the measuring station and the control center, a LAN is built with optical fiber. The industrial computer acts as client and another computer in the control center acts as the server. In the experiments, the client computer is remotely controlled by the server computer.

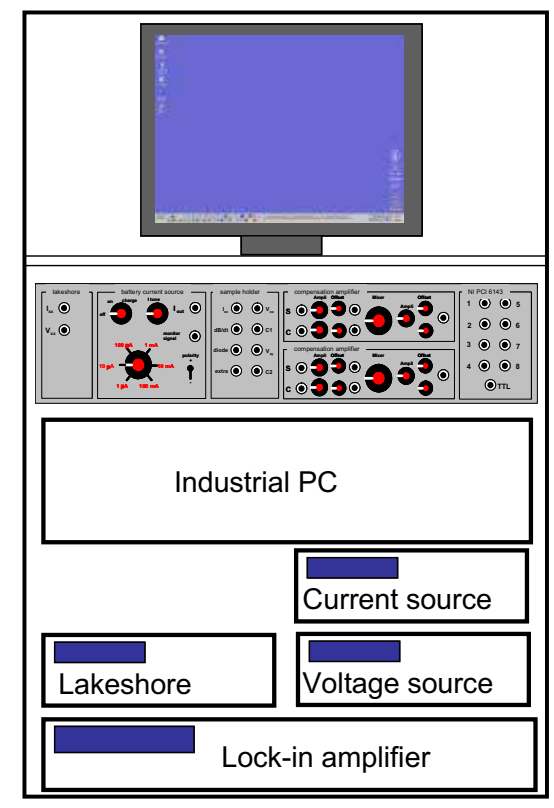

Figure 3. Layout of the data recording system. 


\section{OUTLOOK}

The development of a $12 \mathrm{MJ}$ high energy density capacitor bank is planned to obtain magnetic fields in the 50-80 T range with pulse duration from $15 \mathrm{~ms}$ to $200 \mathrm{~ms}$. In addition to monolithic magnets, a dual coil system will be developed that is energized by two capacitor banks to generate $80 \mathrm{~T}$ with a short rise time. A $50 \mathrm{~T}$ long pulse magnet with $100 \mathrm{~ms}$ flat top will be developed; this will be energized by the $100 \mathrm{MJ}, 100 \mathrm{MVA}$ pulsed AC generator. When the new facility is finished, there will be 7 measuring stations available for scientific research.

\section{ACKNOWLEDGMENT}

We want to express our gratitude to the financial support of Chinese Ministry of Education. The support of the bilateral research project Flanders-China Bil 02/01 is also gratefully acknowledged.

\section{REFERENCES}

1. Peng T et al., "Advanced Numerical Simulation of Pulsed Magnets with a Finite Element Method," Measurement Science and Technology, Vol. 16, 2005, pp. 562568

2. Witte $\mathrm{H}$ et al. "Pulsed Magnets-Advances in Coil Design Using Finite Element Analysis" IEEE Transactions on Applied Superconductivity, Vol. 16, 2006, pp. 1680-1683

3. Herlach F et al. "Experimental and Theoretical Analysis of the Heat Distribution in Pulsed Magnets," IEEE Transactions on Applied Superconductivity, Vol. 16, 2006, pp. 1689-1691

4. Herlach F et al. "Element of Pulsed Magnet Design," in RHMF 2006

5. Vanacken J et al., "Pulsed Magnet Design Software," Physica B, Vol. 294-295, 2001, pp. 674-678

6. Li L et al. "Magnetic and thermal diffusion in pulsed high-field magnets," J. Phys.D: Appl. Phys. Vol. 31, 1998, pp. 1320-1328

7. European project DeNUF Design study for next generation pulsed magnet user facilities

8. Li L et al. "Deformation Analysis of Pulsed Magnets with Internal and External Reinforcement," Measurement Science and Technology, Vol. 6, 1995, pp. 1035-1042 